Gynäkologische Endokrinologie 2011 · 9:85-86 DOI 10.1007/s10304-010-0395-5

Online publiziert: 20. April 2011

(c) Springer-Verlag 2011

\author{
M.K. Bohlmann ${ }^{1} \cdot$ K. Diedrich ${ }^{1} \cdot$ M. Ludwig ${ }^{2}$ \\ ${ }^{1}$ Klinik für Frauenheilkunde und Geburtshilfe, Universitätsklinikum Schleswig-Holstein, \\ Campus Lübeck \\ 2 Zentrum für Endokrinologie, Kinderwunsch und Pränatale Medizin im Barkhof, \\ Hamburg
}

\title{
Differenzialdiagnostik und Therapieoptionen der Hyperandrogenämie
}

tracht gezogen werden. Durch eine spezifische Therapie betroffener Frauen kann eine Zyklusregulierung erreicht sowie gegebenenfalls eine pränatale Virilisierung weiblicher Nachkommen effektiv verhindert werden. V. Deckwart aus Lübeck hat sich in ihrem Beitrag der Bedeutung des Late-onset-AGS in der Kinderwunschbehandlung angenommen. klassische adrenogenitale Syndrom (AGS) zugrunde, so ist eine frühzeitige und lebenslange Therapie der Neugeborenen notwendig, die an diesem Krankheitsbild leiden. C. Bullmann und T. Minnemann aus Hamburg haben für Sie die therapeutischen Aspekte dieser normalerweise im Rahmen des Neugeborenenscreening diagnostizierten Erkrankung zusammengestellt.

Eine ganz andere klinische Bedeutung kommt dem Late-onset-AGS zu, das sich gewöhnlich erst nach der Pubertät manifestiert. Insbesondere bei Kinderwunschpatientinnen mit Zyklusstörungen sowie klinischen Anzeichen einer Hyperandrogenämie ist eine weitergehende endokrine Abklärung notwendig. Die Mutation der 21-Hydroxylase, die einem Late-onsetAGS meist zugrunde liegt, ist dabei nicht nur von Bedeutung für die betroffene $\mathrm{Pa}$ tientin selbst, sondern unter Umständen auch für deren Nachkommen. Wurde eine Mutation dieses Schlüsselenzyms des Androgenstoffwechsels bei einer Patientin nachgewiesen, sollte aufgrund der hohen Heterozygotenprävalenz (1:40) auch beim Partner eine genetische Abklärung in Be-

\section{(2) Beim polyzystischen Ovarsyndrom müssen auch Langzeitfolgen beachtet werden}

Das polyzystische Ovarsyndrom (PCOS) zählt mit einer Prävalenz von etwa 10\% zu den häufigsten gynäkologischen Erkrankungen von Frauen im reproduktiven Alter. Neben der klassischen Trias von Zyklusstörung, Hyperandrogenämie und sonographisch nachgewiesenen polyzystischen Ovarien sind bei diesen Patientinnen insbesondere auch Langzeitfolgen wie die Entwicklung eines Diabetes mellitus oder vaskulären Hypertonus und das erhöhte Risiko eines Endometriumkarzinoms zu beachten. Neben einer Optimierung des Lebensstils kann auch eine Basistherapie indiziert sein. L.-M. Wallwiener aus der Heidelberger Arbeitsgruppe um T. Strowitzki und B. Toth stellt Ihnen die aktuellen Therapieoptionen bei PCOSPatientinnen mit oder ohne Kinderwunsch vor.

PCOS-Patientinnen sind häufig übergewichtig, was zudem oft mit einer
Insulinresistenz einhergeht. Mit einer geeigneten Kostform bietet sich ein therapeutischer Ansatz, der deutlich über eine alleinige Gewichtsreduktion hinausgehen kann. N. Worm aus München und Koautoren präsentieren Ihnen eine spezifische Ernährungsstrategie für PCOS-Patientinnen. Als Ökotrophologen kommt ihnen dabei eine besondere Expertise zu.

Eine sich rasch entwickelnde, ausgeprägte Virilisierung, ein neu auftretender Hirsutismus in der Postmenopause oder eine ausgeprägte Hyperandrogenämie müssen uns bei Frauen auch an die Möglichkeit eines androgenproduzierenden Tumors denken lassen. Eine laborchemische Hormondiagnostik hat zeitnah zu erfolgen. Bei fortbestehendem Tumorverdacht sind endokrine Stimulations- und Suppressionstests, transabdominale oder vaginale Sonographie, Computertomographie, Magnetresonanztomographie und spezifische nuklearmedizinische Verfahren die möglichen weiteren Schritte zur genauen Lokalisationsdiagnostik. F. Hoellen aus Lübeck hat diese für Sie zusammengefasst und in ihrer Relevanz bewertet. Des Weiteren hat sie eine Übersicht der verschiedenen Subformen androgenproduzierender Tumoren erstellt.

Frauen mit Hirsutismus, einem männlichen Verteilungsmuster der Terminalhaare, weisen oft einen hohen Leidensdruck auf. Neben herkömmlichen Verfahren zur Enthaarung finden bei diesen Patientinnen auch lasertherapeutische Ansätze sowie die topische Applikation 
spezifischer Enzyminhibitoren Anwendung. Die Hamburger Dermatologen A. Gust und V. Steinkraus präsentieren Ihnen diese Optionen der kosmetischen Hirsutismustherapie und gehen dabei insbesondere auf mögliche Komplikationen ein.

Wir hoffen, Ihnen mit dieser Mischung von Themen aus verschiedenen Fachbereichen ein interessantes Heft bieten zu können.

Herzlichst,

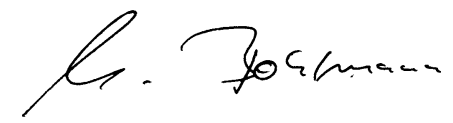

PD Dr. M.K. Bohlmann

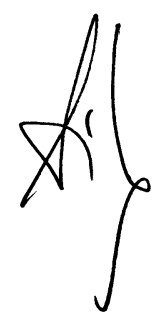

Prof. Dr. M. Ludwig

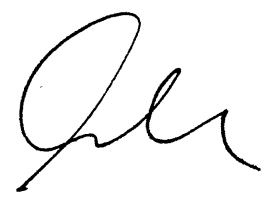

Prof. Dr. K. Diedrich

\section{Korrespondenzadresse}

\section{PD Dr. M.K. Bohlmann}

Klinik für Frauenheilkunde und Geburtshilfe, Universitätsklinikum Schleswig-Holstein, Campus Lübeck Ratzeburger Allee 160, 23538 Lübeck michael.bohlmann@uk-sh.de

\section{Themenübersicht}

Die „Gynäkologische Endokrinologie“ bietet Ihnen umfassende und aktuelle Beiträge zu interessanten Themenschwerpunkten aus allen Bereichen der gynäkologischen Endokrinologie und Reproduktionsmedizin.

Wir haben die Jahrgänge 2010/2011 im Überblick für Sie zusammengestellt:

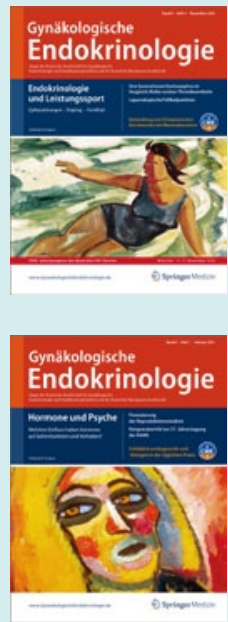

\section{0}

- Heft 1/10 Onkologische Endokrinologie

- Heft 2/10 Mythen in der Reproduktionsmedizin

- Heft 3/10 Ovar: von der Fetalperiode bis zur Menopause

- Heft 4/10 Endokrinologie und Leistungssport

2011

- Heft 1/11 Hormone und Psyche

- Heft 2/11 Differenzialdiagnostik und Therapieoptionen der Hyperandrogenämie

- Heft 3/11 Menopause und Frauengesundheit

- Heft 4/11 Reproduktionsmedizin

Bestellen Sie einzelne Ausgaben oder abonnieren Sie die Zeitschrift zum Preis von EURO 125,- pro Jahr (zzgl. Versandkosten, Ermäßigung für Ärzte in Aus- und Weiterbildung und Fachgesellschaften)! Unser Kundenservice steht Ihnen für Fragen und Informationen gerne zur Verfügung:

Springer-Verlag, Kundenservice Zeitschriften

Haberstrasse 7, 69126 Heidelberg

Tel: + 496221/345-4303, Fax: +496221/345-4229

E-Mail: subscriptions@springer.com

\section{Beiträge auf einen Blick}

Abonennten haben online Zugriff auf alle Beiträge im elektronischen Volltextarchiv unter www.GynaekologischeEndokrinologie.de.

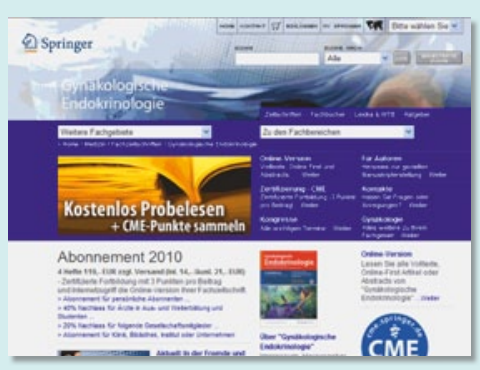

Ins Volltextarchiv gelangen Sie von der Startseite über den Navigationspunkt: "Online Version".

Nach einer einmaligen Registrierung können Sie die Beiträge als pdf oder in der html-Version abrufen. 